\title{
THE ROLE OF SPHINGOSINE 1-PHOSPHATE IN INFLAMMATION AND CANCER
}

\author{
Nigel J. Pyne ${ }^{1}$, Jan Ohotski ${ }^{1}$, Robert Bittman ${ }^{2}$ and Susan Pyne ${ }^{1}$
}

${ }^{1}$ Cell Biology Research Group, Strathclyde Institute of Pharmacy and Biomedical Science, University of Strathclyde, 161 Cathedral Street, Glasgow G4 0RE, UK. ${ }^{2}$ Department of Chemistry and Biochemistry, Queens College of the City University of New York, Flushing, New York 11367-1597, USA.

ABSTRACT--The enzymes that catalyse the formation of bioactive sphingolipid, sphingosine 1phosphate, sphingosine kinase 1 and 2 , are predictive markers in inflammatory diseases and cancer as evidenced by data from patients, knockout mice and the use of available molecular and chemical inhibitors. Thus, there is a compelling case for therapeutic targeting of sphingosine kinase. In addition, there are several examples of functional interaction between sphingosine 1-phosphate receptors and sphingosine kinase 1 that can drive malicious amplification loops that promote cancer cell growth. These novel aspects of sphingosine 1-phosphate pathobiology are reviewed herein.

The lipid sphingosine 1-phosphate (S1P) is a key regulator of cell growth, survival, invasion, lymphocyte trafficking, vascular integrity and cytokine production, and plays a central role in inflammatory disease and cancer. S1P is formed by phosphorylation of sphingosine, catalysed by sphingosine kinases 1 and 2 (SK1 and SK2) that differ in their biochemical properties, sub-cellular localization, and function (Pyne and Pyne, 2011). S1P is cleaved by S1P lyase to produce trans-2-hexadecenal and phosphoethanolamine. S1P can also be dephosphorylated by S1P phosphatases to recycle into sphingolipids (Pyne and Pyne, 2011). S1P is an agonist of S1P-specific G-protein coupled receptors, termed $\mathrm{S}_{1} \mathrm{P}_{1-5}$, and also binds to intracellular protein targets, such as histone deacetylase 1 and 2 (HDAC1/2, which regulate gene expression) (for review see Pyne and Pyne, 2011). 


\section{S1P and inflammation}

S1P has been linked to regulating the production of pro-inflammatory cytokines, Toll-like receptor signalling and inflammation. Inflammatory mediators might regulate SK1 via an ERK-catalysed phosphorylation of SK1 on S225; this promotes SK1 translocation to the plasma membrane, where it catalyzes the formation of S1P (Pitson et al., 2005). S1P is then transported out of the cell and/or partitions in the plasma-membrane, where it binds to a family of G-protein-coupled receptors $\left(\mathrm{S}_{1} \mathrm{P}_{1-5}\right)$ to induce a multitude of cell responses. This process has been termed "inside-out signalling"' (Takabe et al., 2008). For instance, this mechanism leads to the expression of adhesion molecules, such as vascular cell adhesion molecule (VCAM) and intercellular adhesion molecule (ICAM) in response to tumour necrosis factor alpha (TNF- $\alpha)$ (Xia et al., 1998). SK1 and S1P are also required for TNF- $\alpha$-induced cyclooxygenase 2 (COX2) and prostaglandin E2 (PGE2) production (Pettus et al., 2003). TNF- $\alpha$ also activates SK1, resulting in S1P accumulation in synoviocytes from rheumatoid arthritis patients, which promotes proliferation and cytokine production from these cells (Kitano et al., 2006). These studies, along with many others, have firmly established that TNF- $\alpha$ signalling leads to activation of the SK1/S1P pathway. Intracellular S1P also binds to and stimulates TRAF2 E3-ligase activity resulting in the lysine63-linked polyubiquitination (signalling ubiquitination) of receptor interacting protein 1 (RIP1) (Alvarez et al., 2010). Polyubiquitinated RIP1 acts as a scaffold for the recruitment and phosphorylation of the IKK complex, followed by activation of $\mathrm{NK} \kappa \mathrm{B}$, which is a key player in inflammation.

Lipopolysaaccharide (LPS) stimulation of Toll-Like Receptor-4 (TLR-4) in macrophages increases SK1 mRNA and enzyme activity, resulting in generation of S1P and induction of COX2 (Hammad et al., 2008). However, while siRNA knockdown of SK1 has no effect on LPS-stimulated inflammation, it does block TNF $\alpha$-stimulated inflammation (Hammad et al., 2008) and protects against LPS-stimulated apoptosis of macrophages, demonstrating dual and distinct roles of SK1 in TNF and LPS inflammatory pathways. A 
role for SK1 in LPS signaling was also observed by Wu et al., (2004) who demonstrated that SK inhibitors or siRNA knockdown of SK1 expression reduces LPS-stimulation of ERK-1/2 and p38 MAPK, and enhances LPS-stimulated JNK activation. In addition, over-expression of a dominant negative kinase inactive SK1 mutant blocks LPS-stimulated Elk-1 and NFKB transcriptional activity (Wu et al., 2004). SK1 mRNA and protein are also increased in LPS-activated microglia (Nayak et al., 2010), thereby implicating the SK1/S1P pathway in neuroinflammation. In addition, the transactivation of S1PR by IgE receptor, Fc3RI is necessary for mast cell degranulation and migration (Jolly et al., 2004), whereas cytokine production from mast cells requires SK2 (Oskeritzian et al., 2004).

There is also substantial evidence for a role of SK1 in animal models of inflammation. For instance, S1P levels are increased in wild-type mice with dextran sulphate-induced colitis, but not in $S K 1^{-/-}$mice, which have reduced local and systemic inflammation (Snider et al., 2009). In murine models of inflammatory disease, such as arthritis, chemical inhibition or siRNA knockdown of SK1 results in reduced inflammation (Lai et al., 2008). A role for SK1 is also evident in patients with rheumatoid arthritis, where there is an increase in S1P levels in the synovium (Alvarez et al., 2007). Other inflammatory signalling molecules, such as IL-1 $\beta$, IFN- $\gamma$ and IgE (Alvarez et al., 2007) also activate SK1 thereby providing additional compelling evidence for a role of SK1/S1P in the inflammatory response. Treatment of inflammatory disease using drugs that modulate S1P biology is already evident in the clinic, providing proof of concept that SK1 and S1P are bone fide targets for therapeutic intervention. The synthetic sphingosine analogue and pro-drug FTY720 (fingolimod, Gilenya ${ }^{\mathrm{TM}}$ ), is used as an oral drug for the treatment of multiple sclerosis (MS). FTY720 is taken up by cells, phosphorylated by SK2 and released as FTY720 phosphate. FTY720-phosphate binds to and induces functional antagonism of the S1P receptor, $\mathrm{S}_{1} \mathrm{P}_{1}$, to induce lymphopenia, thereby inhibiting attack within the central nervous system by $\mathrm{T}$ lymphocytes (Brinkmann et al., 2010). Egress of T-lymphocytes from the lymph nodes requires an S1P 
gradient. In addition, FTY720 reduces pro-inflammatory cytokine release by astrocytes in multiple sclerosis (Choi et al., 2011). This is significant because FTY720 is also a SK1 inhibitor (Tonelli et al., 2010) and might suggest a role for SK1 in MS.

S1PR modulation with FTY720 has also been studied in mouse models of asthma. In this case, FTY720treated mice previously sensitized to ovalbumin (OVA) exhibit reduced bronchial constriction and eosinophilia compared with vehicle-treated mice. FTY720 also prevents the migration of dendritic cells and inhibits T-lymphocyte activation (Idzko et al., 2006), and reduces hindpaw edema, joint destruction, and lymphocyte invasion in collagen-induced arthritis (CIA) and adjuvant-induced arthritis (AA) (Wang et al., 2007; Matsuura et al., 2000). Furthermore, disease progression in CIA is reduced in mice treated with SK1 siRNA, while SK2 siRNA increases disease incidence and severity. In addition, the SK2 selective inhibitor, ABC294640 (see below) reduces TLR4 expression, NFkB activation, TNF- $\alpha$, IL-1 $\beta$ and CXCL-10 mRNA formation, ICAM1 expression and infiltration of monocytes/macrophages and neutrophils (Liu et al., 2012). ABC294640 also reduces CD4+ lymphocyte infiltration and IFN- $\gamma$ production (Liu et al., 2012). In this regard, murine SK2 interacts with the IL-12 receptor $\beta 1$, to promote IL-12 stimulated formation of IFN- $\gamma$ (Yoshimoto et al., 2003). Additional evidence supporting a proinflammatory role for SK2 is the finding that adenoviral over-expression of wild-type SK2 enhances LPSinduced lung injury (Wadgaonkar et al., 2009).

These studies, along with many others, have firmly established a key role for SK1 and SK2 in inflammatory disease, and as such, both enzymes are targets for novel anti-inflammatory therapeutics. However, there is also evidence that SK2 may have an anti-inflammatory role, and therefore there is a need to provide clarity. For instance, SK2-deficient MCF-7 breast cancer cells exhibit increased levels of pro-inflammatory cytokines and decreased levels of anti-inflammatory IL-10, which is associated with a 
decrease in tumour growth (Samy et al., 2007). Moreover, when T-lymphocyte-deficient C.B-17 scid mice were injected with $S K 2^{-/-}$T-lymphocytes, pro-inflammatory cytokines levels increased and intestinal inflammation was more severe compared with mice receiving wild-type T-lymphocytes. This appears due to enhanced IL-2 responsiveness and increased expression of activated phosphorylated STAT5 (Samy et al., 2007).

\section{Current advances in the identification of SK1 and SK2 inhibitors}

Multiple options exist for the development of SK1 inhibitors. Enzyme kinetic studies show that many of the SK inhibitors that have been developed are competitive with sphingosine, but allosteric inhibitors are also an exciting option (see below). The Pyne lab recently showed that SK1 contains an allosteric site (Lim et al., 2011a) and that replacement of the amino group in $(S)$-FTY720-vinylphosphonate with an azido group, changes this compound from an allosteric inhibitor to an activator of SK1 (Liu et al., 2013). The first inhibitors of SK1 were sphingosine analogs, such as D,L-threo-dihydrosphingosine (DHS) which has a $\mathrm{Ki}$ of $\sim 5 \mu \mathrm{M}$ (Pyne and Pyne, 2011). N,N-Dimethylsphingosine (DMS), which was originally identified as a PKC inhibitor, also inhibits both SK isoforms. The first inhibitor that was highly selective for SK1 was the water-soluble sphingosine analog, SK1-I (Ki $\sim 10 \mu \mathrm{M})$ (Paugh et al., 2008). Currently, the most potent nanomolar SK1-selective inhibitor is PF-543 (Schnute et al., 2012). Using a synthetic route from 4-octylphenethyl alcohol, we have produced a series of FTY720-like analogues that are SK1selective inhibitors (e.g. RB-005) (Baek et al., 2013). ABC294640 (French et al., 2010) and (R)-FTY720 methylether (ROMe) (Lim et al., 2011b) are SK2-selective inhibitors with $\mathrm{K}_{\mathrm{i}}$ values $\sim 10 \mu \mathrm{M}$. With the very recent resolution of the crystal structure of SK1 (Wang et al., 2103), it may now be possible to understand the mechanisms of action of these compounds and their selectivity for SK1. 


\section{Allosteric inhibitors}

Allosteric inhibitors and activators might bind to unique allosteric sites in SK1 or alternatively, might induce cooperative effects with bound substrate. For instance, $(S)$-FTY720 vinylphosphonate contains a hydroxyl group that can form a hydrogen bond with A339 via a water molecule. Therefore, $(S)$-FTY720 vinylphosphonate is expected to overlap the binding mode of sphingosine and should act as a competitive inhibitor with sphingosine. However, our kinetic study revealed that $(S)$-FTY720 vinylphosphonate is an uncompetitive inhibitor with sphingosine (Lim et al., 2011a), which requires that $(S)$-FTY720 vinylphosphonate binds to the SK1-sphingosine complex and not to the free enzyme. We and others have also reported that SK1 is a dimer (Kihara et al., 2006; Lim et al., 2011a); therefore, binding of sphingosine to the catalytic site in one of the monomers could promote binding of $(S)$-FTY720 vinylphosphonate to the catalytic site in the second monomer. In this manner, the binding of substrate and inhibitor molecules do not compete and inhibition is uncompetitive. Once $(S)$-FTY720 vinylphosphonate has bound to the second monomer it might reduce the phosphorylation of sphingosine by displacing the critical amino-acid residues away from sphingosine, thereby preventing deprotonation of the primary hydroxy group of sphingosine, which is required for catalysis.

\section{Allosteric activators}

The azido derivative of (S)-FTY720 vinylphosphonate is an activator of SK1 (Liu et al., 2013), as is a regioisomer of FTY720 (Lim et al., 2011a), suggesting that the amino group in (S)-FTY720 vinylphosphonate is essential for allosteric regulation and that these activators bind in a manner where there is overlap in the catalytic pocket. One possibility is that the binding of an activator in one of the monomers promotes binding of sphingosine to the catalytic site in the second monomer. In this manner, overall catalysis is more efficient compared with occupation of both sites with sphingosine. One would predict that as the activator concentration is increased, the activator should also bind in a manner that 
overlaps the catalytic pocket of the second monomer. Under these conditions, both catalytic sites will be occupied with activator, thereby precluding binding of sphingosine. Under these conditions, the enzyme will effectively be inhibited. Indeed, we found that two azido analogues of (S)-FTY720 vinylphosphonate exhibit a bell-shaped concentration response curve, with activation of SK1 at low concentrations and inhibition at high concentrations (Liu et al., 2013). Allosteric inhibitors offer the prospect of conferring exquisite specificity for SK1. Furthermore, crystallisation studies and modelling might enable development of allosteric inhibitors with very high affinity, improving on current inhibitors that only have micromolar potency. Our studies indicate that SK1 contains an auto-inhibitory domain which is either stabilised in the 'on-state' by inhibitors or stabilised in the 'off-state' by activators (Lim et al., 2011a).

\section{Use of inhibitors to interrogate TLR4- and TNF- $\alpha$-dependent signaling}

We propose that TNF- $\alpha$-dependent activation of SK1 results in S1P which can be released from cells to act on S1P receptors and thereby promotes STAT3-mediated IL-6 formation (Fig. 1). Indeed, binding of $\mathrm{S} 1 \mathrm{P}$ to $\mathrm{S}_{1} \mathrm{P}_{1}$ receptor has been shown to promote $\mathrm{NK \kappa B}$ and STAT3 activation and IL-6 formation in colitis-induced cancer, which is driven by aberrant macrophage pathology (Liang et al., 2013; Pyne and Pyne, 2013). The role of SK/S1PR 'inside-out' signalling can be tested by assessing the effect of S1PR antagonists and siRNA knockdown (compared with scrambled siRNA) of S1PR on TNF- $\alpha$ - and S1Pstimulated NFkB activation, STAT3 phosphorylation and IL-6 formation. Alternatively, S1P might stimulate TRAF2/receptor interacting protein 1 (RIP1) signalling and IKK activation. S1P derived from SK2 might enhance LPS signalling by binding to intracellular targets in the MyD88-dependent pathway to amplify signal transmission from TLR4, expression of which is regulated by SK2 (Liu et al., 2012). 


\section{S1P and cancer}

Role of SK1 in inflammation-linked cancer

There is substantial evidence of a role for SK1 in numerous cancers (Pyne and Pyne, 2010; Pyne et al., 2012). There is increased expression of SK1 in stomach, lung, brain, colon, kidney and breast cancers and non-Hodgkins lymphoma (Pyne and Pyne, 2010). In addition, high expression of SK1 in patient tumours, including breast cancer and astrocytoma grade 4, is associated with poor clinical prognosis (Van Brocklyn et al., 2005; Watson et al., 2010; Ohotoski et al., 2012). There is also a link between inflammation and cancer in terms of the participation of SK1. Thus, high expression of SK1 is associated with metasatic colon cancer (Kawamori et al., 2009). Indeed, the colon carcinogen, azoxymethane increases SK1 expression and S1P levels in tumours (Kawamori et al., 2009). Moreover, SK1 knockout mice subjected to azoxymethane develop significantly less aberrant crypt foci formation and exhibit reduced colon cancer progression (Kawamori et al., 2009). Indeed, silencing of SK1 reduces COX2 and PGE2 production in HT-29 colon cancer cells (Kawamori et al., 2006). S1P also promotes a malicious amplification loop involving $\mathrm{S}_{1} \mathrm{P}_{1}$, NFKB, IL-6 and STAT3 to promote colitis-induced colon cancer (Liang et al., 2013). Moreover, Liang et al. (2013) have recently demonstrated that FTY720 ablates colitis-induced colon cancer via inhibition/down-regulation of SK1 expression.

\section{Role of SK1 in regulating cell survival}

We have shown that the treatment of androgen-sensitive LNCaP cells with the SK inhibitor, SKi ((2-(phydroxyanilino)-4-(p-chlorophenyl)thiazole) (French et al., 2003)), induces the proteasomal degradation of SK1 (Loveridge et al., 2010). SKi also promotes formation of pro-apoptotic diadenosine 5, $,{ }^{,},{ }_{-}-P^{1}, P^{3}-$ triphosphate (Ap3A) (Watson et al., 2013). Tryptophanyl-tRNA synthetase uses ATP, ADP and tryptophan to produce Ap3A, which binds to the effector, FHIT (fragile histidine triad protein) tumour suppressor gene product (Vartanian et al., 1997; Fischer and McLennen, 2008) and is removed by FHIT 
hydrolase activity. Growth suppression induced by FHIT involves up-regulation of the cell cycle regulator and cyclin-dependent kinase inhibitor, p2 ${ }^{\text {waf1 }}$ (Sard et al., 1999). FHIT has also been shown to interact with ferridoxin reductase to produce reactive oxygen species (ROS) and to thereby induce apoptosis of cancer cells (Trapasso et al., 2008). Therefore, since Ap3A accumulates when SK1 is degraded by the proteasome in response to SK inhibitors, we propose that SK1 limits the tryptophanyl-tRNA synthetasecatalysed formation of Ap3A or promotes FHIT hydrolase activity. This might prevent FHIT-dependent activation of ferridoxin reductase and ROS formation and this leads to cancer cell survival. The finding that SK inhibitor increases Ap3A levels are significant as over-expression of FHIT induces apoptosis in cancer cells with FHIT gene abnormalities (Ji et al., 1999). Moreover, Fhit knockout mice are predisposed to tumour development, and Fhit gene therapy reduces tumour burden (Pichiorri et al., 2008). In addition, down regulation of FHIT protein is linked with extra-prostatic extension and Gleason score > 8 in prostate cancer and breast cancer. Evidence strongly suggests the involvement of germ-line variations of FHIT in prostate cancer risk. Restoration of wild-type FHIT in 3p14.2-deficient human lung cancer cells also inhibits cell growth and induces apoptosis (Deng et al., 2007). Thus, the SK1-FHIT axis may be one mechanism whereby SK1 regulates cell survival.

\section{Role of SK1 in regulating amplification loops}

We have reported that S1P promotes translocation of SK1 to the plasma-membrane of MCF-7 breast

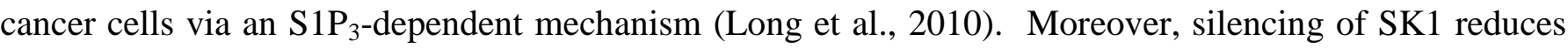
$\mathrm{S}_{1} \mathrm{P}_{3}$ expression and results in a decrease in $\mathrm{S} 1 \mathrm{P} / \mathrm{S} 1 \mathrm{P}_{3}$-stimulated ERK-1/2 activation and migration of MCF-7 cells (Long et al., 2010). Therefore, SK1 regulates responsiveness of these cancer cells to S1P by increasing $\mathrm{S}_{1} \mathrm{P}_{3}$ expression levels. This might produce a positive amplification loop of $\mathrm{S}_{1} \mathrm{P}_{3}$-mediated invasive signalling in breast cancer cells (Fig. 2). 


\section{Role of SK1 in regulating the Warburg effect}

We have also demonstrated that SK1 inhibitors, such as SKi, induce the ubiquitin-proteasomal degradation of SK1 by activating the proteasome (Loveridge et al., 2010). We have also performed the first metabolomic analysis in prostate cancer cells treated with $\mathrm{SKi}$, under conditions where SK1 is degraded by the proteasome (Loveridge et al., 2010). The findings suggest that SK1 promotes the Warburg effect (Fig. 3), which is an essential survival pathway for cancer cells to obtain ATP for biosynthetic and catabolic metabolism (Watson et al., 2013). Treatment of androgen-sensitive prostate cancer cells with the SK1 inhibitor, SKi, increases glycolytic metabolites and $(R)$-S-lactoyl-glutathione levels (Watson et al., 2013); the latter is formed from methylglyoxal (a highly reactive glycolytic byproduct that is apoptotic in prostate cancer cells). Treatment of androgen-sensitive prostate cancer cells with SKi also increases glucose 6-phosphate utilisation by the pentose phosphate pathway in order to provide the anti-oxidant NADPH to counter oxidative stress responses (Watson et al., 2013). The glutathione system uses NADPH to recycle glutathione (GSH). In this case, the protection afforded by NADPH toward oxidative stress is inadequate and the prostate cancer cells appear to be overwhelmed by ROS; this might explain the subsequent apoptotic response. SKi also promotes the proteasomal degradation of c-Myc, a consequence of ceramide-dependent activation of the proteasome, which might account for the effect of this inhibitor on the Warburg effect (Watson et al., 2013). Indeed, c-Myc is a master transcriptional regulator of the glycolytic pathway (Osthus et al., 2000; Shim et al., 1997). A reduction in c-Myc level is also found in polyps of ApcMin/ $S k 1^{-/}$mice (Kohno et al., 2006), thereby confirming a regulatory link between SK1 and c-Myc expression.

\section{Nuclear $\mathrm{SlP}_{2}$ receptor signalling and interaction with $S K 1 / S K 2$ and $S R C$}

High estrogen receptor positive $\left(\mathrm{ER}^{+}\right)$tumour expression of nuclear $\mathrm{SRC}$ and nuclear $\mathrm{S}_{1} \mathrm{P}_{2}$ in patients is associated with longer disease-specific survival time and is therefore protective against mortality (Ohotski 
et al., 2013). In addition, tumours with high levels of nuclear $\mathrm{S}_{1} \mathrm{P}_{2}$ receptor have significantly reduced levels of nuclear SK1 (which is linked with poor prognosis). This suggests the presence of an active translocation mechanism for SK1 that is regulated by $\mathrm{S}_{1} \mathrm{P}_{2}$ and which might account for its protective action in cancer patients (Ohotski et al., 2013). We have demonstrated that treatment of triple negative MDA-MB-231 breast cancer cells with the SK1 inhibitor, SKi, promotes the accumulation of ectopically expressed wild type $\mathrm{S}_{1} \mathrm{P}_{2}$ along with tyrosine phosphorylated (Y416) Src (non-receptor tyrosine kinase) in the nucleus of these cells (Fig. 4). Our proposed model is that S1P prevents the nuclear localisation of $\mathrm{S} 1 \mathrm{P}_{2}$ and Y416 Src. Therefore, inhibition of SK1 with pharmacological inhibitors reduces S1P levels, enabling the translocation of $\mathrm{S}_{1} \mathrm{P}_{2}-\mathrm{Y} 416 \mathrm{Src}$ to the nucleus. Indeed, treatment of these cells with $\mathrm{S} 1 \mathrm{P}$ reduces the $\mathrm{SKi}$-induced nuclear translocation of $\mathrm{S}_{1} \mathrm{P}_{2}-\mathrm{Y} 416 \mathrm{Src}$. Therefore, SK1 might protect against apoptosis of breast cancer cells by preventing nuclear $\mathrm{S}_{1} \mathrm{P}_{2}-\mathrm{Y} 416 \mathrm{Src}$ localization and induced expression of apoptotic genes.

\section{Conclusion}

Although the crystal structure of SK1 has been resolved, this has been achieved with SKi, a low-affinity SK1 inhibitor. The functional interaction with a high-affinity inhibitor is necessary to determine the complete structural architecture of the catalytic site. This may lead to the development of better SK1 inhibitors that can be used to induce apoptosis of cancer cells and to enhance anti-inflammatory activity. However, a recent study showed that a nanomolar inhibitor of SK1 (PF-543) failed to kill cancer cells in vitro (Schnute et al., 2012). This observation has led to serious questions about whether SK1 is a viable therapeutic target in cancer, despite a wealth of evidence for a critical role of SK1 in cancer cell survival, growth, transformation, metastasis and neovascularisation. The high binding affinity of PF-543 for SK1 might indicate the possibility that this compound might also bind to other enzymes that use sphingosine as a substrate e.g. ceramide synthases. Inhibition of ceramide synthase is likely to neutralize the effect of 
inhibiting SK1 activity on cell growth and survival by preventing formation of ceramide from sphingosine that is accumulates as a result of inhibiting/down-regulating SK1. Thus, development of more potent SK inhibitors requires binding modalities that confer specificity toward SK over other sphingolipid metabolizing enzymes. This might be essential in order to potentiate killing of cancer cells by selective inhibition of SK1.

\section{REFERENCES}

Alvarez SE, Harikumar KB, Hait NC, Allegood J, Strub GM, Kim EY, et al. Sphingosine 1-phosphate is a missing co-factor for the E3 ligase TRAF2. Nature 2010; 465:1084-1088.

Alvarez SE, Milstien S, Spiegel S. Autocrine and paracrine roles of sphingosine-1-phosphate. Trends Endo Metabol 2007; 18:300-307.

Baek DJ, MacRitchie N, Pyne NJ, Pyne S, Bittman R. The synthesis of selective inhibitors sphingosine kinase 1. Chem Comm 2013; 49:2136-2138.

Brinkmann V, Billich A, Baumruker T, Heining P, Schmouder R, Francis G, et al. Fingolimod (FTY720): discovery and development of an oral drug to treat multiple sclerosis. Nat Rev Drug Discov. 2010; 9: 883-897.

Choi JW, Gardell SE, Herr DR, Rivera R, Lee CW, Noguchi K, et al. FTY720 (fingolimod) efficacy in an animal model of multiple sclerosis requires astrocyte sphingosine 1-phosphate receptor $1\left(\mathrm{~S}_{1} \mathrm{P}_{1}\right)$ modulation. Proc Natl Acad Sci (USA) 2011; 108:751-756.

Deng WG, Nishizaki M, Fang B, Roth JA, Ji L. Induction of apoptosis by tumor suppressor FHIT via death receptor signaling pathway in human lung cancer cells. Biochem Biophys Res Comm 2007; 355:993-999.

Fischer DI, McLennen AG. Correlation of intracellular diadenosine triphosphate (Ap3A) with apoptosis in Fhit-positive HEK 293 cells. Cancer Lett 2008; 259:186-191.

French KJ, Schrecengost RS, Lee BD, Zhuang Y, Smith SN, Eberly JL, et al. Discovery and evaluation 
of inhibitors of human sphingosine kinase. Cancer Res. 2003; 63:5962-5969.

French KJ, Zhuang Y, Maines LW, Gao P, Wang W, Beljanski V, et al. Pharmacology and anti-tumour activity of ABC294640, a selective inhibitor of sphingosine kinase-2. J Pharmacol Exp Ther 2010; 333:129-139.

Hammad SM, Crellin HG, Wu BX, Melton J, Anelli V, Obeid LM. Dual and distinct roles for sphingosine kinase 1 and sphingosine 1 phosphate in the response to inflammatory stimuli in RAW macrophages. Prostaglandins Other Lipid Mediat 2008; 85:107-114.

Idzko M, Hammad H, van Nimwegen M, Kool M, Müller T, Soullié T, et al. Local application of FTY720 to the lung abrogates experimental asthma by altering dendritic cell function. J Clin Invest 2006; 116:2935-2944

Ji L, Fang B, Yen N, Fong K, Minna JD, Roth JA. Induction of apoptosis and inhibition of tumorigenicity and tumor growth by adenovirus vector-mediated Fragile Histidine Triad (FHIT) gene overexpression. Cancer Res 1999; 59:3333-3339.

Jolly PS, Bektas M, Olivera A, Gonzalez-Espinosa C, Proia RL, Rivera J, et al. Transactivation of sphingosine-1-phosphate receptors by FcepsilonRI triggering is required for normal mast cell degranulation and chemotaxis. J Exp Med 2004; 199:959-970.

Kawamori T, Osta W, Johnson KR, Pettus BJ, Bielawski J, Tanaka T, et al. Sphingosine kinase 1 is upregulated in colon carcinogenesis. FASEB J 2006; 20:386-388.

Kawamori T, Kaneshiro T, Okumura M, Maalouf S, Uflacker A, Bielawski J, et al. Role for sphingosine kinase 1 in colon carcinogenesis. FASEB J 2009; 23:405-414.

Kihara A, Anada Y, Igarashi Y. Mouse sphingosine kinase isoforms SPHK1a and SPHK1b differ in enzymatic traits including stability, localization, modification, and oligomerization. J Biol Chem 2006; 281: 4532-4539.

Kitano M, Hla T, Sekiguchi M, Kawahito Y, Yoshimura R, Miyazawa K, et al. Sphingosine 1- 
phosphate/sphingosine 1-phosphate receptor 1 signaling in rheumatoid synovium: regulation of synovial proliferation and inflammatory gene expression. Arthritis Rheum 2006; 54:742-753.

Kohno M, Momoi M, Oo ML, Paik JH, Lee YM, Venkataraman K et al. Intracellular role for sphingosine kinase 1 in intestinal adenoma cell proliferation. Mol Cell Biol. 2006; 26:7211-7223. Lai WQ, Irwan AW, Goh HH, Howe HS, Yu DT, Valle-Oñate R, McInnes IB, Melendez AJ, Leung BP. Anti-inflammatory effects of sphingosine kinase modulation in inflammatory arthritis. J Immunol 2008; 181:8010-8017.

Liang J, Nagahashi M, Kim EY, Harikumar KB, Yamada A, Huang WC, et al Sphingosine-1-phosphate links persistent STAT3 activation, chronic intestinal inflammation, and development of colitisassociated cancer. Cancer Cell 2013; 23:107-120.

Lim KG, Tonelli F, Li Z, Lu X, Bittman R, Pyne S, Pyne NJ. FTY720 analogues as sphingosine kinase 1 inhibitors: Enzyme inhibition kinetics, allosterism, proteasomal degradation and actin rearrangement in MCF-7 breast cancer cells. J Biol Chem 2011a; 286:18633-18640.

Lim KG, Sun C, Bittman R, Pyne NJ, Pyne S. $(R)$-FTY720 methyl ether is a specific sphingosine kinase 2 inhibitor: effect on sphingosine kinase 2 expression in HEK 293 cells and actin rearrangement and survival of MCF-7 breast cancer cells. Cell Signal 2011b; 23:1590-1595.

Liu Q, Rehman H, Shi Y, Krishnasamy Y, Lemasters JJ, Smith CD, Zhong Z. Inhibition of sphingosine kinase-2 suppresses inflammation and attenuates graft injury after liver transplantation in rats. PLoS One 2012; 7:e41834.

Liu Z, MacRitchie N, Pyne S, Pyne NJ, Bittman R. Synthesis of (S)-FTY720 vinylphosphonate analogues and evaluation of their potential as sphingosine kinase 1 inhibitors and activators. Bioorg Med Chem 2013; 21:25013-2510.

Long JS, Edwards J, Watson C, Tovey S, Mair K, Schiff R, et al. Sphingosine kinase 1 induces tolerance to human Epidermal growth factor receptor 2 and prevents formation of a migratory 
phenotype in response to sphingosine 1-phosphate in estrogen receptor positive breast cancer cells. Mol Cell Biol 2010: 30:3827-3841.

Loveridge C, Tonelli F, Leclercq TM, Lim KG, Long JS, Berdyshev E, et al. The sphingosine kinase 1 inhibitor 2-(p-hydroxyanilino)-4-(p-chlorophenyl)thiazole induces proteasomal degradation of sphingosine kinase 1 in mammalian cells. J Biol Chem 2010; 285:38841-38852.

Matsuura M, Imayoshi T, Okumoto T. Effect of FTY720, a novel immunosuppressant, on adjuvant- and collagen-induced arthritis in rats. Int J Immunopharmacol 2000; 22:323-331.

Nayak D, Huo Y, Kwang WX, Pushparaj PN, Kumar SD, Ling EA, et al. Sphingosine kinase 1 regulates the expression of proinflammatory cytokines and nitric oxide in activated microglia. Neuroscience 2010; 166:132-144.

Ohotski J, Long JS, Orange C, Elsberger B, Mallon E, Doughty J, et al. Expression of sphingosine 1phosphate receptor 4 and sphingosine kinase 1 is associated with outcome in oestrogen receptornegative breast cancer. Br J Cancer. 2012;106:1453-1459.

Ohotski J, Edwards J, Elsberger B, Watson C, Orange C, Mallon E, et al. Identification of novel functional and spatial associations between sphingosine kinase 1, sphingosine 1-phosphate receptors and other signaling proteins that affect prognostic outcome in estrogen receptor-positive breast cancer. Int J Cancer 2013; 132:605-616

Oskeritzian CA, Alvarez SE, Hait NC, Price MM, Milstien S, Spiegel S. Distinct roles of sphingosine kinases 1 and 2 in human mast-cell functions. Blood 2004; 111:4193-4200.

Osthus RC, Shim H, Kim S, Li Q, Reddy R, Mukherjee M, et al.. Deregulation of glucose transporter 1 and glycolytic gene expression by c-Myc. J Biol Chem. 2000; 275:21797-21800.

Paugh SW, Paugh BS, Rahmani M, Kapitonov D, Almenara JA, Kordula T, et al. A selective sphingosine kinase 1 inhibitor integrates multiple molecular therapeutic targets in human leukemia. Blood 2008; 112:1382-1391. 
Pettus BJ, Bielawski J, Porcelli AM, Reames DL, Johnson KR, Morrow J, et al. The sphingosine kinase 1/sphingosine-1-phosphate pathway mediates COX-2 induction and PGE2 production in response to TNF- $\alpha$. FASEB J 2003; 17:1411-1421.

Pichiorri F, Palumbo T, Suh SS, Okamura H, Trapasso F, Ishii H, et al. Fhit tumor suppressor: guardian of the preneoplastic genome. Future Oncol 2008; 4:815-824.

Pitson SM, Xia P, Leclercq TM, Moretti PA, Zebol JR, Lynn HE, et al. Phosphorylation-dependent translocation of sphingosine kinase to the plasma membrane drives its oncogenic signalling. J Exp Med 2005; 201:49-54.

Pyne NJ, Pyne S. Sphingosine 1 phosphate and cancer. Nature Rev Cancer 2010; 10:489-503.

Pyne NJ, Pyne S. Sphingosine 1-phosphate is the missing link between inflammation and colon cancer. Cancer Cell 2013; 23:5-7.

Pyne S, Pyne NJ. Translational aspects of sphingosine 1-phosphate biology. Trends Mol Med 2011; $17: 463-472$.

Pyne NJ, Tonelli F, Lim KG, Long S, Edwards J, Pyne S. Targeting sphingosine kinase 1 in cancer. Advances in Biological Regulation 2012; 52:31-38.

Samy ET, Meyer CA, Caplazi P, Langrish CL, Lora JM, Bluethmann H, et al. Cutting edge: Modulation of intestinal autoimmunity and IL-2 signaling by sphingosine kinase 2 independent of sphingosine 1-phosphate. J Immunol 2007;179:5644-5648.

Sard L, Accornero P, Tornielli S, Delia D, Bunone G, Campiglio M, et al. Tumor-suppressor gene FHIT is involved in the regulation of apoptosis and in cell cycle control. Proc Natl Acad Sci (USA) 1999; 96:8489-8492.

Schnute ME, McReynolds MD, Kasten T, Yates M, Jerome G, Rains JW, et al. Modulation of cellular S1P levels with a novel, potent and specific inhibitor of sphingosine kinase-1. Biochem J 2012; 444:79-88. 
Shim H, Dolde C, Lewis BC, Wu CS, Dang G, Jungmann RA, et al. c-Myc transactivation of LDH-A: implications for tumor metabolism and growth. Proc Natl Acad Sci (USA) 1997; 94:6658-6663.

Snider AJ, Kawamori T, Bradshaw SG, Orr KA, Gilkeson GS, Hannun YA et al. A role for sphingosine kinase 1 in dextran sulfate sodium-induced colitis. FASEB J 2009; 23:143-152.

Takabe K, Paugh SW, Milstien S, Spiegel S. 'Inside-out' signalling of sphingosine-1-phosphate: therapeutic targets. Pharmacol Rev 2008; 60:181-195.

Tonelli F, Lim KG, Loveridge C, Long J, Pitson SM, Tigyi G, et al. FTY720 and (S)-FTY720 vinylphosphonate inhibit sphingosine kinase 1 and promote its proteasomal degradation in human pulmonary artery smooth muscle, breast cancer and androgen-independent prostate cancer cells. Cell Signal 2010; 22:1536-42.

Trapasso F, Pichiorri F, Gaspari M, Palumbo T, Aqeilan RI, Gaudio E, et al. Fhit interaction with ferredoxin reductase triggers generation of reactive oxygen species and apoptosis of cancer cells. J Biol Chem 2008; 283:13736-13744.

Vartanian A, Prudovsky I, Suzuki H, Dal Pra I, Kisselev L. Opposite effect of cell differentiation and apoptosis on AP3A/AP4A ratio in human cell cultures. FEBS Lett 1997; 415:160-162.

Wadgaonkar R, Patel V, Grinkina N, Romano C, Liu J, Zhao Y, et al. Differential regulation of sphingosine kinases 1 and 2 in lung injury. Am J Physiol Lung Cell Mol Physiol 2009: 296; L603L613.

Wang Z, Min X, Xiao SH, Johnstone S, Romanow W, Meininger D, et al. Molecular basis of sphingosine kinase 1 substrate recognition and catalysis. Structure 2013; 21:798-809.

Wang F, Tan W, Guo D, He S. Reduction of CD4 positive T cells and improvement of pathological changes of collagen-induced arthritis by FTY720. Eur J Pharmacol 2007; 573:230-240.

Watson C, Long JS, Orange C, Tannahill CL, Mallon E, McGlynn LM, et al. High expression of sphingosine 1-phosphate receptors, $\mathrm{S}_{1} \mathrm{P}_{1}$ and $\mathrm{S}_{1} \mathrm{P}_{3}$, sphingosine kinase 1, and extracellular signal- 
regulated kinase-1/2 is associated with development of Tamoxifen resistance in estrogen receptorpositive breast cancer patients. Am J Pathology 2010; 177:2205-15.

Watson DG, Tonelli F, Alossaimi M, Williamson L, Chan E, Gorshkova I, et al. The roles of sphingosine kinases 1 and 2 in regulating the Warburg effect in prostate cancer cells. Cell Signal $2013 ; 25: 1011-1017$.

Wu W, Mosteller RD, Broek D. Sphingosine kinase protects lipopolysaccharide-activated macrophages from apoptosis. Mol Cell Biol 2004; 24:7359-7369.

Van Brocklyn JR, Jackson CA, Pearl DK, Kotur MS, Snyder PJ, Prior TW. Sphingosine kinase-1 expression correlates with poor survival of patients with glioblastoma multiforme: role of sphingosine kinase isoforms in growth of glioblastoma cell lines. J Neuropathol Exp Neurol 2005; 64:695-05.

Xia P, Gamble JR, Rye KA, Wang L, Hii CS, Cockerill P, et al. Tumor necrosis factor-alpha induces adhesion molecule expression through the sphingosine kinase pathway. Proc Natl Acad Sci (USA) $1998 ; 95: 14196-14201$.

Yoshimoto T, Furuhata M, Kamiya S, Hisada M, Miyaji H, Magami Y, et al. Positive modulation of IL12 signaling by sphingosine kinase 2 associating with the IL-12 receptor beta 1 cytoplasmic region. J Immunol 2003; 171:1352-1359.

\section{Figure legends}

Fig. 1. Schematic showing the possible role of TNF- $\alpha$ activated $\mathrm{SK} 1$ in $\mathrm{S}_{1} \mathrm{P}_{1}$ receptor-mediated activation of NFKB, STAT3 and IL-6 formation and inflammation

Fig. 2. Schematic showing the SK1-S1P $\mathrm{P}_{3}$ amplification loop in $\mathrm{ER}^{+} \mathrm{MCF}-7$ breast cancer cells that might drive invasiveness and metastasis.

Fig. 3. Schematic summarizing the role of SK1 in inhibiting proteasomal activity thereby maintaining cMyc expression levels. c-Myc is a master transcriptional regulator of the glycolytic enzymes that drives 
the Warburg effect and limits flux through the pentose phosphate pathway. SK1 also limits the formation of Ap3A and oxidative stress.

Fig. 4. Schematic summarizing how the $\mathrm{SK} 1$ inhibitor, $\mathrm{SKi}$, promotes nuclear localization of $\mathrm{S}_{1} \mathrm{P}_{2}-\mathrm{Y}_{416}$ Src in ER ${ }^{-}$MDA-MB-231 cells that might contribute to the effect of these inhibitors on cancer cell growth, DNA synthesis and apoptosis. 
Fig. 1

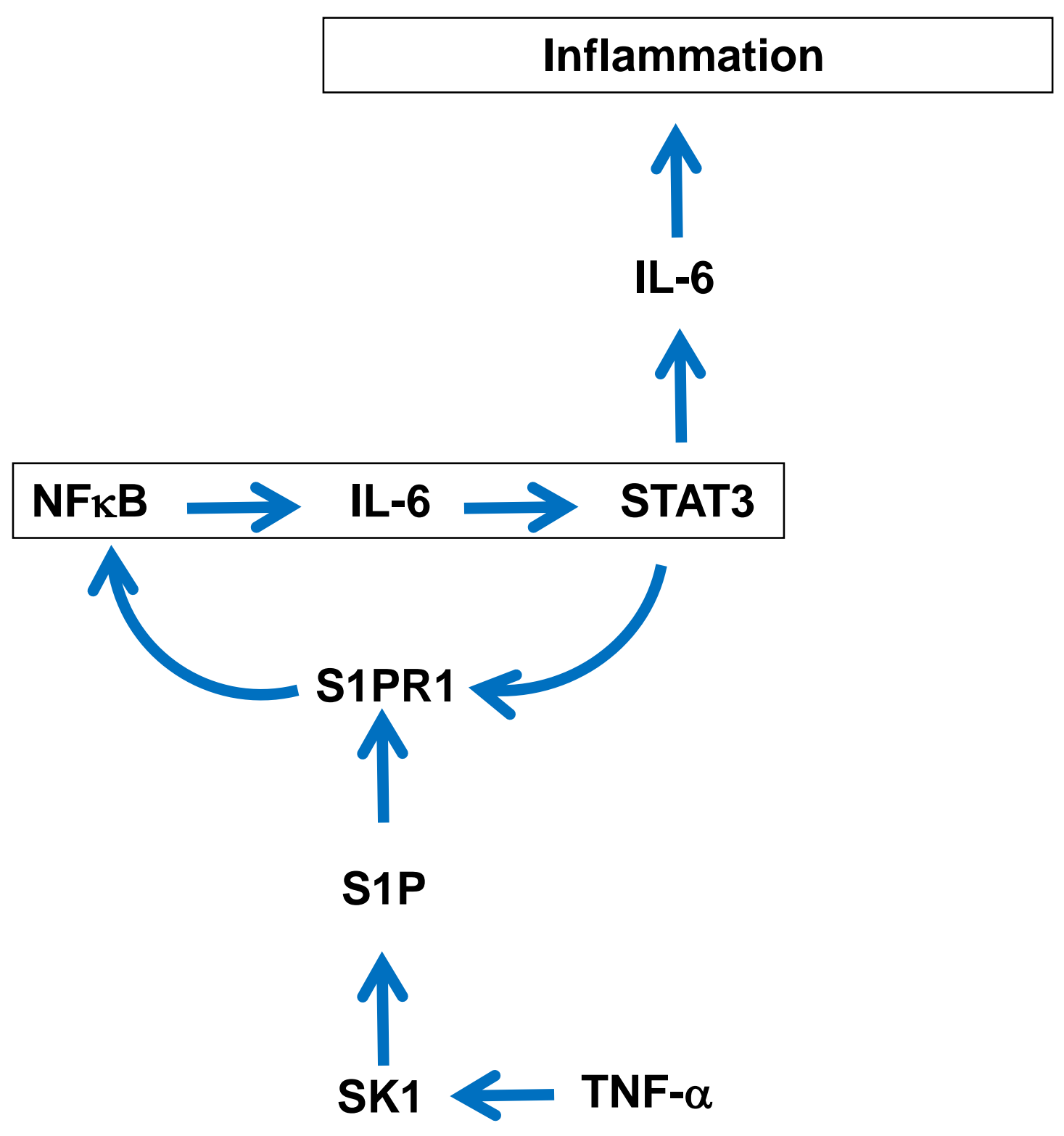


Fig. 2

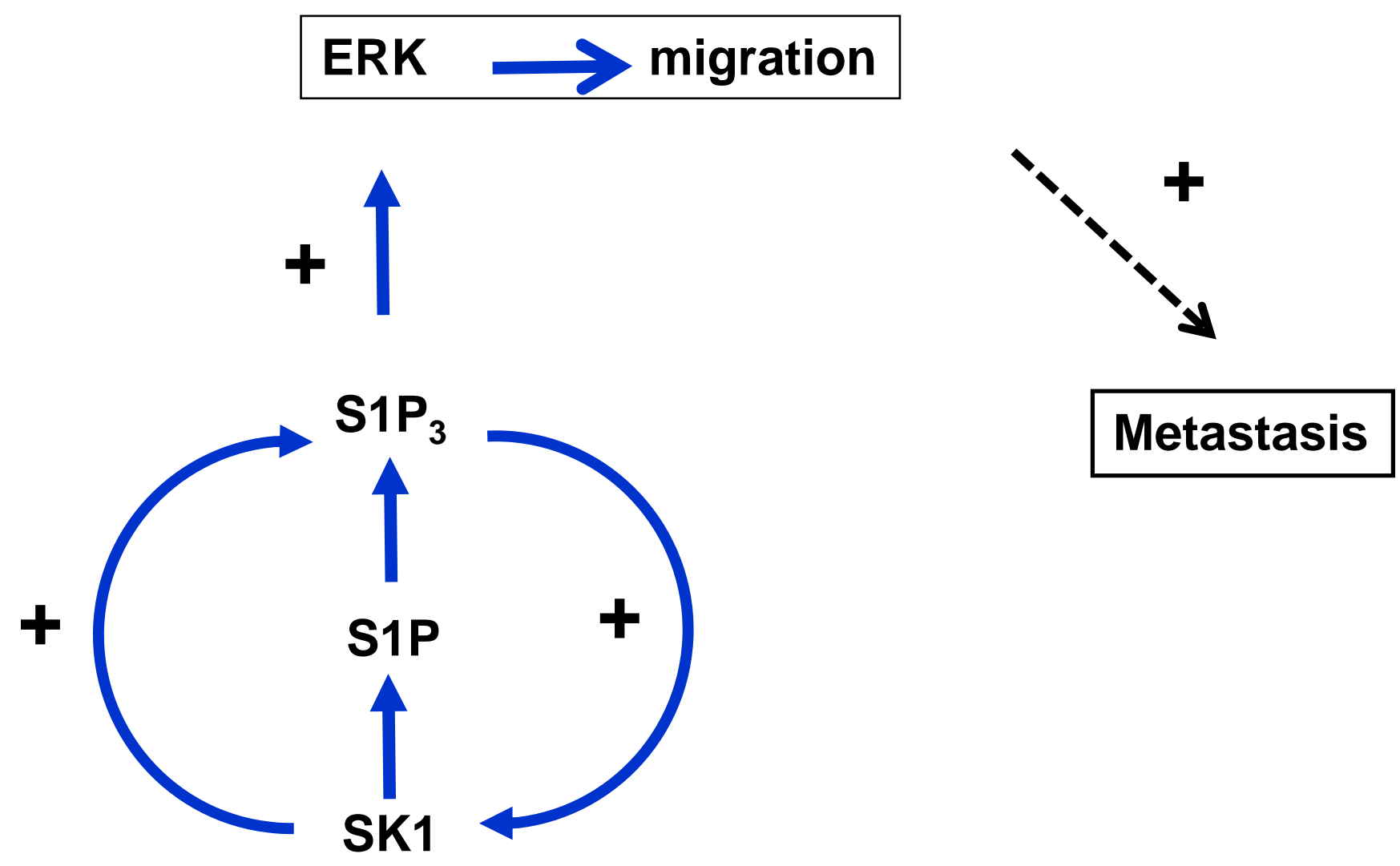


Fig. 3

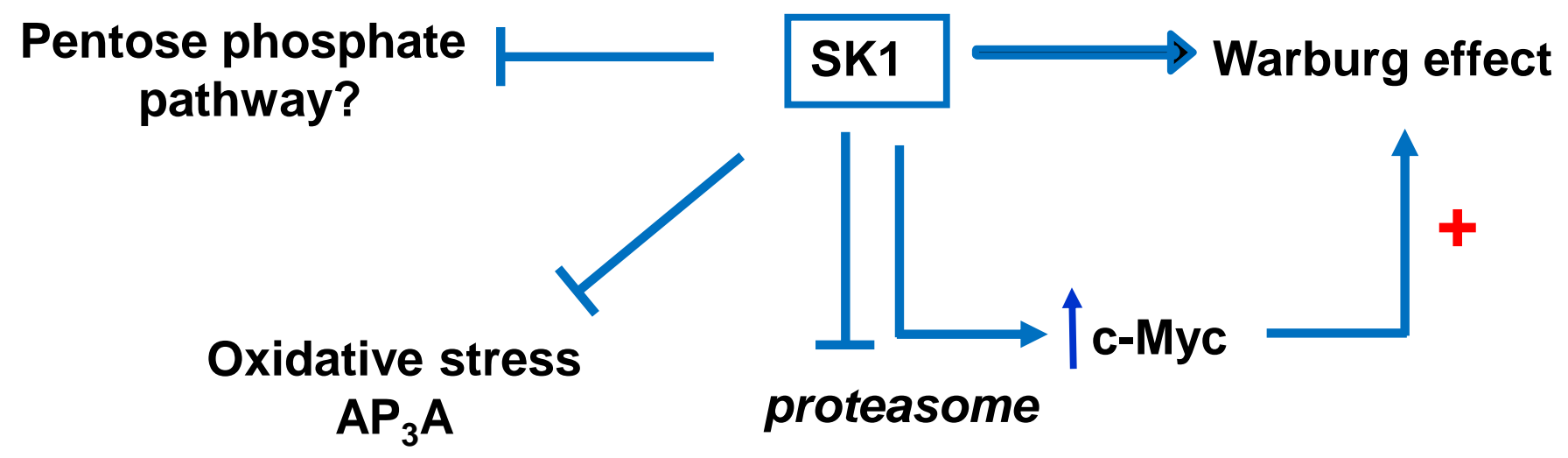


Fig. 4

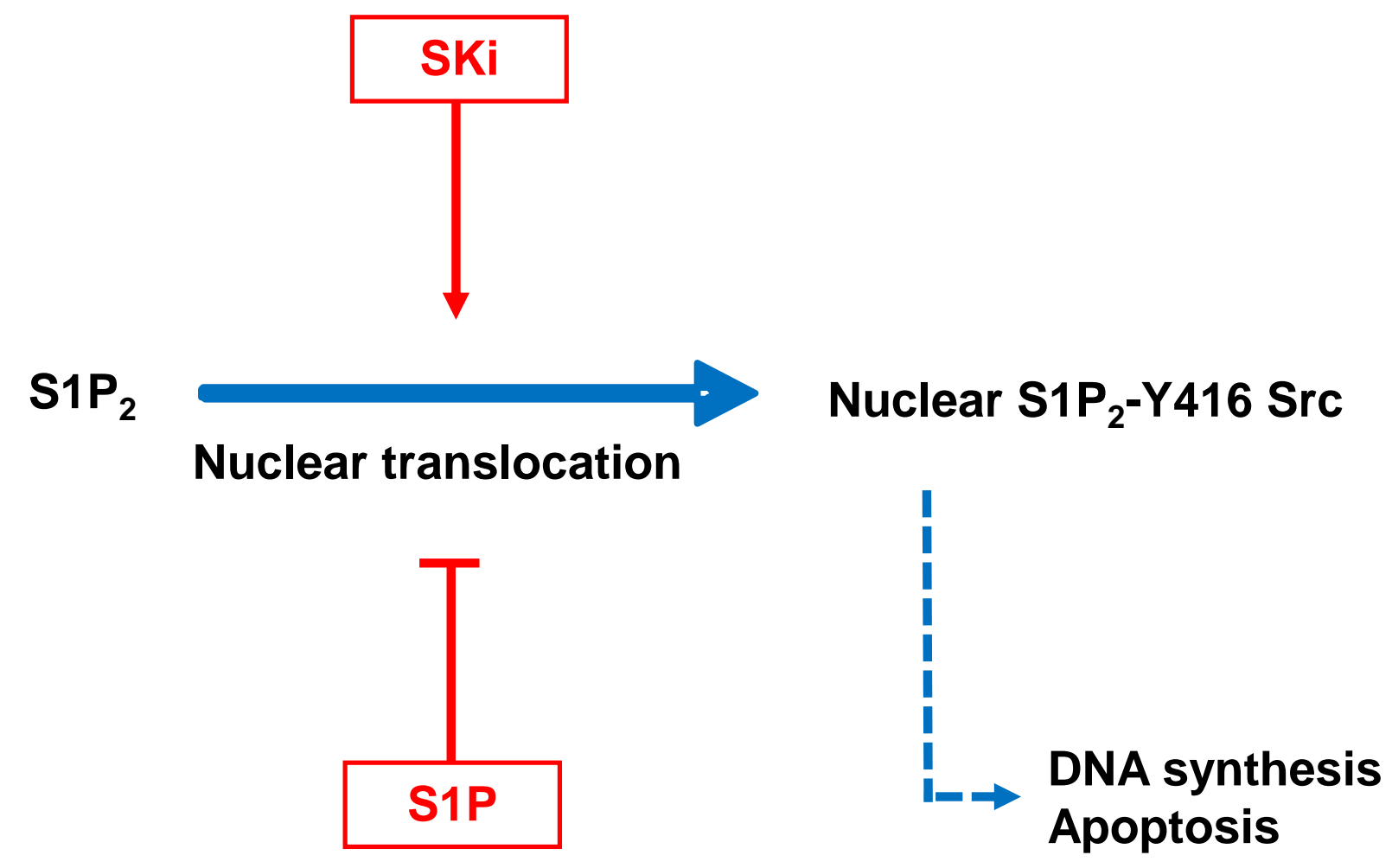

